\title{
Prognostic value of right ventricular dilatation in patients with low-risk pulmonary embolism
}

\author{
Benoit Côté ${ }^{1}$, David Jiménez ${ }^{2}$, Benjamin Planquette $3,4,5,6$, Anne Roche P.4 $^{3,4,5,}$ \\ Jonathan Marey ${ }^{3,4}$, Jean Pastré ${ }^{3,4}$, Guy Meyer ${ }^{3,4,6,7}$ and Olivier Sanchez $z^{3,4,5,6}$ \\ Affiliations: 'Département de Médecine Interne, Hôpital de l'Enfant-Jésus du CHU de Québec, Université \\ Laval, Québec, Canada. ${ }^{2}$ Respiratory Dept, Ramon y Cajal Hospital, Alcala de Henares University, IRYCIS, \\ Madrid, Spain. ${ }^{3}$ Division of Respiratory and Intensive Care Medicine, Hôpital Européen Georges Pompidou, \\ Paris, France. ${ }^{4}$ Université Paris Descartes, Sorbonne Paris Cité, Paris, France. ${ }^{5}$ INSERM UMRS 1140, Paris, \\ France. ${ }^{6}$ F-CRIN, INNOVTE, Saint Etienne, France. ${ }^{7}$ INSERM UMRS 970 and CIC 1418, Paris, France.
}

Correspondence: Benoit Côté, Département de Médecine Interne, Hôpital de l'Enfant-Jésus du CHU de Québec, Université Laval, 1401 18e rue Quebec, Quebec G1J 1Z4, Canada. E-mail: benoit.cote.6Quulaval.ca

@ERSpublications

In PE patients with sPESI $0, \mathrm{MDCT} \mathrm{RV} / \mathrm{LV} \geqslant 0.9$ and $\geqslant 1.0$ are not associated with worse prognosis but higher cut-off values might be http://ow.ly/Jda730guiFz

Cite this article as: Côté B, Jiménez D, Planquette B, et al. Prognostic value of right ventricular dilatation in patients with low-risk pulmonary embolism. Eur Respir J 2017; 50: 1701611 [https://doi.org/10.1183/ 13993003.01611-2017].

ABSTRACT The prognosis of multidetector computed tomography (MDCT) assessed right ventricular dilatation (RVD) is unclear in patients with pulmonary embolism (PE) and a simplified Pulmonary Embolism Severity Index (sPESI) of 0 . We investigated in these patients whether MDCT-assessed RVD, defined by a right to left ventricular ratio $(\mathrm{RV} / \mathrm{LV}) \geqslant 0.9$ or $\geqslant 1.0$, is associated with worse outcomes.

We combined data from three prospective cohorts of patients with PE. The main study outcome was the composite of 30-day all-cause mortality, haemodynamic collapse or recurrent PE in patients with sPESI of 0.

Among 779 patients with a sPESI 0, $420(54 \%)$ and $299(38 \%)$ had a RV/LV $\geqslant 0.9$ and $\geqslant 1.0$ respectively. No difference in primary outcome was observed, $0.95 \%$ (95\% CI $0.31-2.59$ ) versus $0.56 \%$ (95\% CI 0.10 $2.22 ; \mathrm{p}=0.692$ ) and $1.34 \%$ (95\% CI $0.43-3.62)$ versus $0.42 \%$ (95\% CI $0.07-1.67 ; \mathrm{p}=0.211)$ with $\mathrm{RV} / \mathrm{LV} \geqslant 0.9$ and $\geqslant 1.0$ respectively. Increasing the $\mathrm{RV} / \mathrm{LV}$ threshold to $\geqslant 1.1$, the outcome occurred more often in patients with RVD $(2.12 \%, 95 \%$ CI $0.68-5.68$ versus $0.34 \%$, 95\% CI $0.06-1.36$; $\mathrm{p}=0.033)$.

MDCT RV/LV ratio of $\geqslant 0.9$ and $\geqslant 1.0$ in sPESI 0 patients is frequent but not associated with a worse prognosis but higher cut-off values might be associated with worse outcome in these patients.

\footnotetext{
This article has supplementary material available from erj.ersjournals.com

Received: June 222017 | Accepted after revision: Sept 082017

Conflict of interest: Disclosures can be found alongside this article at erj.ersjournals.com

Copyright @ERS 2017
} 


\section{Introduction}

The European Society of Cardiology (ESC) recommends stratifying normotensive pulmonary embolism (PE) patients according to their risk of death using a combination of clinical risk prediction rules, imaging assessing of right ventricular (RV) function and biomarkers to aid their management [1]. The Pulmonary Embolism Severity Index (PESI) and its simplified version (sPESI) identify patients with a low 30-day risk of mortality and complications [2-4]. However, it is unclear whether low-risk patients should receive additional testing to improve their risk stratification. The ESC guidelines do not recommend additional testing of low-risk patients. Nevertheless, multidetector computed tomography (MDCT), which is currently used to confirm PE in most patients, can detect RV dilatation (RVD). Clinicians are thus often faced with MDCT-assessed RVD in low-risk patients [5]. MDCT-assessed RVD is associated with a small increase in the risk of death in patients with acute PE, but whether this holds true for low-risk patients is still a matter of debate $[6,7]$. The ESC guidelines suggest upgrading low-risk sPESI patients with RVD from the low to intermediate-low-risk class, making them potentially ineligible for outpatient management [1]. Other data suggest that high N-terminal pro-brain natriuretic peptide (NT pro-BNP) values in patients with a negative Hestia rule are not associated with an increased risk of PE-related complications [8]. Therefore, we aimed to test whether RVD assessed by MDCT is associated with an increased risk of adverse outcomes in patients with sPESI of 0 .

\section{Methods \\ Source study characteristics}

We combined individual patient data from three prospective cohorts of consecutive patients with symptomatic, normotensive and objectively confirmed PE $[9,10]$. These databases contain right to left ventricular ratios (RV/LV ratio) measured by MDCT, information to calculate the sPESI score and 30-day follow-up data.

The first cohort was the Prognostic Factors for Pulmonary Embolism (PREP) study conducted in 11 hospitals in France, Belgium and Switzerland [9]. A total of 592 consecutive patients aged $>18$ years with symptomatic and objectively confirmed PE were included in this study between January 2006 and May 2007. For the present analysis, 41 patients were excluded, because of cardiogenic shock at inclusion, and 22 haemodynamically stable patients were excluded because they received fibrinolytic treatment. A total of 529 clinically stable patients receiving anticoagulant treatment in this study were included in the present study.

The second cohort was the PROgnosTic valuE of Computed Tomography scan in haemodynamically stable patients with acute symptomatic pulmonary embolism (PROTECT) study. This was a prospective, multicentre, observational study involving the emergency departments of 12 hospitals in Spain between January 1, 2009, and May 31, 2011 [10]. A total of 848 haemodynamically stable patients aged $\geqslant 18$ years with an objectively confirmed PE by MDCT were included in this study.

The third source of data included in the present study is derived from a prospective registry of consecutive patients admitted to our department for acute PE. This currently ongoing registry includes consecutive patients with PE included between May 2010 and November 2016 in a single academic centre. This registry was approved by the local ethics committee, which waived the need for signed informed consent for observational studies, according to French regulations (IRB number 1922081-2 February 2016). Among the 1066 patients included in this cohort, 18 were excluded because they received fibrinolytic treatment. Thus, a total of 1048 adults receiving anticoagulation treatment for clinically stable PE were included in the present study.

\section{Development of an individual patient database}

We contacted the principal investigators of each eligible database to explain the aim of the study and they agreed to share their database. Databases were transferred to a central location and a single pooled database was developed by one of the investigators (B. Côté).

\section{Population}

The study population comprised consecutive patients, with an objectively confirmed symptomatic normotensive (i.e. systolic blood pressure $\geqslant 90 \mathrm{mmHg}$ ) PE and a SPESI score of 0 , who were treated with curative anticoagulation and without fibrinolytic treatment.

The sPESI score was calculated retrospectively for each patient, with 1 point given for each of the following: age $>80$ years, oxygen saturation $<90 \%$, systolic blood pressure $<100 \mathrm{mmHg}$, heart rate $\geqslant 110$ beats per minute, history of cancer, history of cardiopulmonary diseases [3]. Missing data were assumed to be normal. 
Measurement of the RV/LV ratio was performed at the valvular plane of the two-dimensional axial transverse plan. RV/LV ratios were provided in the original database of the two previously published studies and were measured by one of the investigators blinded for the outcome in the prospective registry. We defined RVD according to two different RV/LV ratios assessed by MDCT $(\geqslant 0.9$ and $\geqslant 1.0)$ that have been previously associated with a negative outcome in other studies $[6,7,11]$. Then, patients were separated into two groups according to their MDCT RV/LV ratio.

\section{Outcomes}

The main outcome was 30-day adverse events, defined as a composite of all-cause mortality, symptomatic recurrence of a venous thromboembolism event (VTE) or haemodynamic collapse. Each component of the composite and 30-day PE-related mortality were also analysed individually.

PE-related death was defined as death following objective evidence of PE or sudden unexplained death for which PE could not be ruled out. Haemodynamic collapse was defined as the presence of any of the following: the need for cardiopulmonary resuscitation, systolic blood pressure $<90 \mathrm{mmHg}$ for $>15 \mathrm{~min}$, or the need for inotropic or vasopressor drugs. All symptomatic recurrences had to be objectively confirmed by either the presence of a new intraluminal filling defect or an extension of a previous filling defect by MDCT. All events were independently adjudicated in all three cohorts.

\section{Statistical analysis}

Categorical variables are presented as numbers and percentages. Continuous variables are presented as medians and interquartile ranges. The t-test was used for comparisons of continuous variables and the Fisher exact test or the Chi-squared test were used for comparisons of nominal variables.

The proportions of the 30-day outcome according to the presence or absence of RVD by MDCT using cut-off values of $\geqslant 0.9$ and $\geqslant 1.0$ were compared using the Fisher exact test. Each individual component of the composite and PE-related mortality were analysed in same the way. Results are presented as proportions with 95\% confidence intervals. Receiver operating characteristic (ROC) curve analysis was used to determine the area under the curve (AUC) of the RV/LV ratio concerning the main outcome of the study [12].

All analyses were performed using R 3.3.3 (R foundation, Vienna, Austria).

\section{Results}

\section{Patients}

The three databases included 2425 patients with acute PE, among whom 938 (39\%) had a sPESI of 0 (figure 1). Among them, 159 patients were not included because the MDCT RV/LV ratio was not available or the PE diagnosis was obtained with a ventilation/perfusion scan. Thus, the study population comprised 779 patients with a sPESI of 0 .

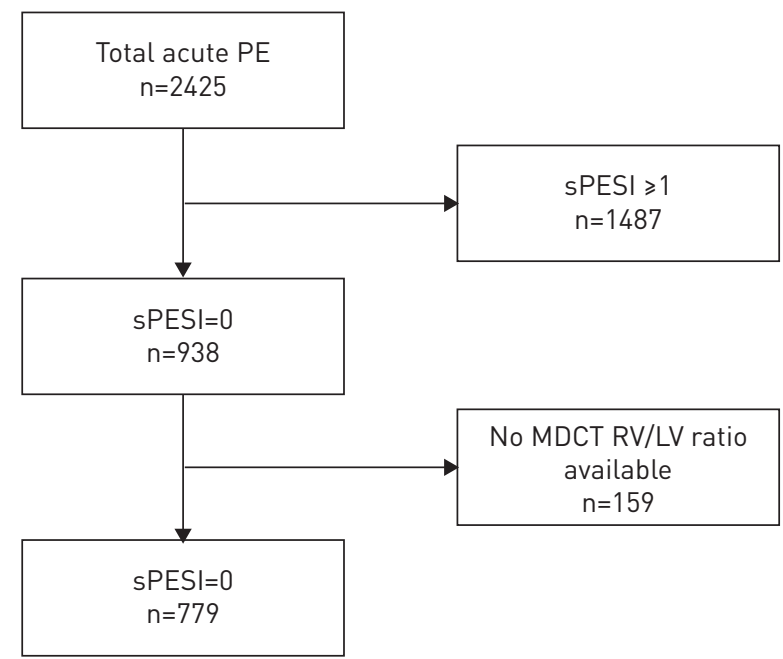

FIGURE 1 Flow chart of study population. PE: pulmonary embolism; sPESI: simplified Pulmonary Embolism Severity Index; MDCT: multidetector computed tomography; RV: right ventricle; LV: left ventricle. 
TABLE 1 Baseline characteristic of the 779 patients with a simplified Pulmonary Embolism Severity Index score of 0 according to a multidetector computed tomography (MDCT) right ventricular dysfunction definition of a right ventricle (RV)/left ventricle (LV) ratio $\geqslant 0.9$

\begin{tabular}{|c|c|c|c|c|c|}
\hline & Available data & All patients & $\mathrm{RV} / \mathrm{LV}<0.9$ & $\mathrm{RV} / \mathrm{LV} \geqslant 0.9$ & $\mathrm{p}$-value \\
\hline Subjects & & 779 & 359 & 420 & \\
\hline Age years & 779 & $61(44-72)$ & $54(39-68)$ & $65(50-74)$ & $<0.001$ \\
\hline Male sex & 779 & $347(45 \%)$ & $165(46 \%)$ & $182(43 \%)$ & 0.462 \\
\hline \multicolumn{6}{|l|}{ Risk factor $\#$} \\
\hline Previous VTE & 779 & $168(22 \%)$ & $75(21 \%)$ & $93(22 \%)$ & 0.672 \\
\hline Recent surgery" & 779 & $95(12 \%)$ & $47(13 \%)$ & $48(11 \%)$ & 0.550 \\
\hline Immobilisation $^{+}$ & 779 & $67(9 \%)$ & $28(8 \%)$ & $39(9 \%)$ & 0.461 \\
\hline Recent travel ${ }^{\S}$ & 779 & $75(10 \%)$ & $37(10 \%)$ & $38(9 \%)$ & 0.553 \\
\hline Hormonal treatment ${ }^{f}$ & 779 & $94(12 \%)$ & $58(16 \%)$ & $36(9 \%)$ & 0.001 \\
\hline Pregnancy & 779 & $4(0.5 \%)$ & $1(0.3 \%)$ & $3(0.7 \%)$ & 0.396 \\
\hline Post partum & 779 & $3(0.4 \%)$ & $2(0.6 \%)$ & $1(0.2 \%)$ & 0.474 \\
\hline \multicolumn{6}{|l|}{ Symptoms and physical examination } \\
\hline Syncope & 779 & $47(6 \%)$ & $10(3 \%)$ & $37(9 \%)$ & $<0.001$ \\
\hline Heart rate & 777 & $84(74-95)$ & $83(72-94)$ & $84(75-95)$ & 0.350 \\
\hline Systolic blood pressure $\mathrm{mmHg}$ & 779 & $134(121-148)$ & $131(120-146)$ & $135(121-137)$ & 0.131 \\
\hline Respiratory rate $\geqslant 30$ per min & 666 & $59 / 666(9 \%)$ & $28 / 315(9 \%)$ & $31 / 351(9 \%)$ & 0.979 \\
\hline \multicolumn{6}{|l|}{ Imaging } \\
\hline MDCT RV/LV ratio & 779 & $0.91(0.80-1.10)$ & $0.80(0.72-0.84)$ & $1.07(0.97-1.25)$ & $<0.001$ \\
\hline $\begin{array}{l}\text { Data are presented as median } \\
\text { simplified Pulmonary Embolism } \\
\S \text { : plane trip within } 1 \text { month; }^{f} \text { : or }\end{array}$ & $\begin{array}{l}\text { range) unless } \\
\text { ex score of } 0 \\
\text { ive or hormon }\end{array}$ & $\begin{array}{l}\text { ise stated. VT } \\
\text { derlying canc } \\
\text { py for menopa }\end{array}$ & $\begin{array}{l}\text { Is thromboemb } \\
\text { argery within }\end{array}$ & $\begin{array}{l}{ }^{\#}: \text { by definition } \\
h:{ }^{+}: \geqslant 3 \text { days of }\end{array}$ & $\begin{array}{l}\text { ient with } \\
\text { ilisation; }\end{array}$ \\
\hline
\end{tabular}

The characteristics of the study population are shown in tables 1 and 2. 420 patients (54\%) had a RV/LV ratio $\geqslant 0.9$ by MDCT and $299(38 \%)$ of $\geqslant 1.0$. With both RVD definition, patients with RVD were older and presented more often with syncope.

\section{Outcomes}

The primary outcome occurred in six patients $(0.77 \%$; $95 \%$ CI $0.31-1.76 \%)$ during the 30-day follow-up period (table 3$)$. Three patients died during follow-up $(0.39 \%$; $95 \%$ CI $0.10-1.22 \%)$ and all deaths were due to PE.

Using a RV/LV ratio $\geqslant 0.9$ to define RVD, four patients with RVD (0.95\%; 95\% CI 0.31-2.59\%) reached the primary outcome versus two without RVD $(0.56 \%$; 95\% CI $0.10-2.22 \%$; $=0.692)$. The 30-day mortality rate in patients with RVD was $0.71 \%$ (95\% CI $0.18-2.25 \%$ ), whereas it was $0 \%$ in the group without RVD (95\% CI 0.00-1.32\%; p=0.254).

Using a RV/LV ratio $\geqslant 1.0$ to define RVD, no significant difference was found regarding the primary outcome (1.34\%; 95\% CI $0.43-3.62$ versus $0.42 \%$; $95 \%$ CI $0.07-1.67 ; \mathrm{p}=0.211$ ) (table 4 ). The 30 -day mortality rate in patients with RVD was $1.00 \%$ (95\% CI $0.26-3.15 \%)$ versus $0 \%$ in the group without RVD (95\% CI 0.00-0.99\%; $\mathrm{p}=0.056)$.

The AUC for the RV/LV ratio measured by MDCT concerning the main outcome of the study is presented in figure 2. The AUC was 0.71 (95\% CI 0.45-0.97). MDCT RVD-assessed performance to evaluate the 30-day adverse event composite according to different threshold values is shown in table 5.

\section{Discussion}

Our study investigated whether RVD assessed by MDCT was associated with an increased risk of adverse events in patients with acute normotensive PE and a sPESI score of 0 . We found that a conventional RV/ $\mathrm{LV}$ ratio of $\geqslant 0.9$ or $\geqslant 1.0$ by MDCT was not associated with an increase in our primary outcome, defined as a composite of all-cause mortality, symptomatic VTE recurrence or secondary shock.

The literature presents conflicting data on the prognostic value of an increased RV/LV ratio in patients with normotensive pulmonary embolism $[6,7,10,11,13]$. The RV/LV ratio, measured by transthoracic cardiac echography (TTE), has been associated with a higher risk of mortality in one meta-analysis, whereas RVD assessed by MDCT was not [13]. In the PROTECT study that followed 848 normotensive patients with PE, no association of MDCT-assessed RVD with 30-day all-cause mortality was observed [10]. In contrast, two recent meta-analyses and one recent cohort study reported an increased risk of death 
TABLE 2 Baseline characteristic of the 779 patients with a simplified Pulmonary Embolism Severity Index score of 0 according to a multidetector computed tomography (MDCT) right ventricular dysfunction definition of a right ventricle (RV)/left ventricle (LV) ratio $\geqslant 1.0$

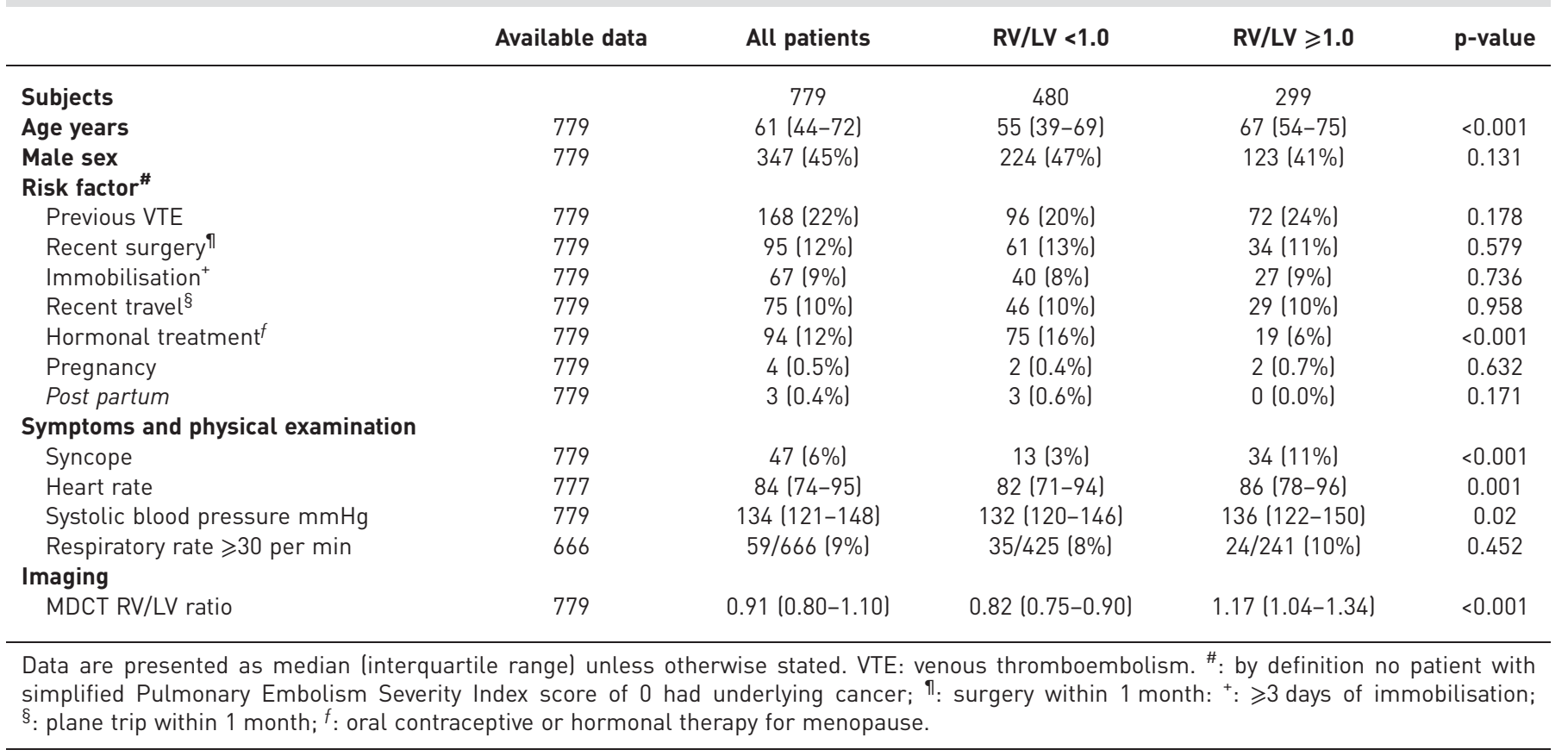

in patients with RVD by MDCT $[6,7,11]$. These studies were performed on patients with varying degrees of clinical severity and did not include only low-risk patients, according to their PESI or sPESI. RVD can be explained not only by acute PE but also by cardiopulmonary comorbidities. Since we included only patients with a sPESI of 0 , all these patients did not have, by definition, any major pulmonary or cardiac comorbidities. As a result, RVD is more probably due to PE in these patients with a sPESI of 0.

We initially planned to define an optimal RV/LV ratio value to predict adverse events using ROC curve analysis. However, since the inferior confidence interval of the AUC was below 0.5, our data did not establish a relationship between the RV/LV ratio measured by MDCT and major complications in patients with PE and a sPESI of 0 . Nevertheless, all three PE-related deaths in the study occurred in patients with a RV/LV ratio higher than 1.2 (supplementary table S2). Moreover, we observed that the rates of PE-related death or shock were significantly higher in patients with $\mathrm{RV} / \mathrm{LV} \geqslant 1.1$ (table 5 and table S3). Although limited by the low number of events, our data suggest that patients with a sPESI of 0 and a higher than recommended cut-off RV/LV value on MDCT seem to be at higher risk for PE-related outcomes. Thus, even with the result of the ROC curve analysis, it seems that a cut-off value of MDCT-assessed RV/LV of $\geqslant 1.1$ could be used to upgrade patients with a sPESI of 0 from the low to the intermediate-low risk class of the ESC. Also, in these patients it may be useful to perform TTE as it has been shown that RVD by TTE using a cut-off RV/LV ratio of 0.9 is associated with a higher risk of adverse events in patients with a low-risk PESI [14]. Of note, TTE was not performed in all patients included in this study; therefore, we

TABLE 3 Study 30-day outcome according to multidetector computed tomography assessed right ventricular dilatation defined by a right ventricle $(\mathrm{RV}) /$ left ventricle $(\mathrm{LV})$ ratio $\geqslant 0.9$

\begin{tabular}{|c|c|c|c|c|}
\hline & All patients & RV/LV $<0.9$ & $\mathrm{RV} / \mathrm{LV} \geqslant 0.9$ & p-value \\
\hline Subjects $\mathrm{n}$ & 779 & 359 & 420 & \\
\hline Primary composite outcomes ${ }^{\#}$ & $6(0.77 \%, 0.31-1.76 \%)$ & $2(0.56 \%, 0.10-2.22 \%)$ & $4(0.95 \%, 0.31-2.59 \%)$ & 0.692 \\
\hline PE mortality & $3(0.39 \%, 0.10-1.22 \%)$ & $0(0 \%, 0.00-1.32 \%)$ & $3(0.71 \%, 0.18-2.25 \%)$ & 0.254 \\
\hline Recurrent PE & $4(0.51 \%, 0.16-1.40 \%)$ & $1(0.28 \%, 0.01-1.79 \%)$ & $3(0.71 \%, 0.18-2.25 \%)$ & 0.629 \\
\hline Haemodynamic collapse & $6(0.77 \%, 0.31-1.76 \%)$ & $2(0.56 \%, 0.10-2.22 \%)$ & $4(0.95 \%, 0.31-2.59 \%)$ & 0.692 \\
\hline
\end{tabular}

Data are presented as $\mathrm{n}(\%, 95 \% \mathrm{Cl})$. PE: pulmonary embolism. ${ }^{\#}$ : one patient could have more than one type of event. 
TABLE 4 Study 30-day outcome according to multidetector computed tomography assessed right ventricular dilatation defined by a right ventricle $(\mathrm{RV}) /$ left ventricle $(\mathrm{LV})$ ratio $\geqslant 1.0$

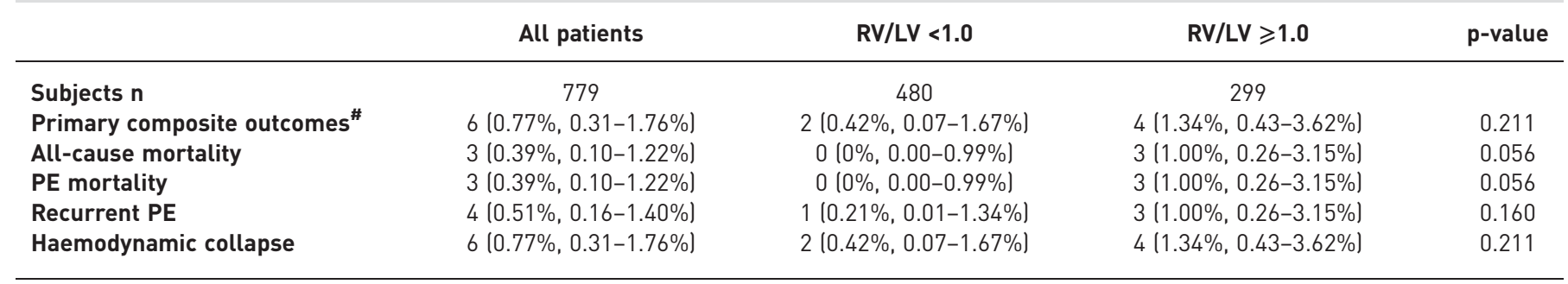

Data are presented as $\mathrm{n}(\%, 95 \% \mathrm{CI})$. PE: pulmonary embolism. ${ }^{\#}$ : one patient could have more than one type of event.

cannot confirm these results. Another advantage of TTE is that it can, in some cases, diagnose right heart thrombi, which are associated with a worse prognosis [15]. This last finding warrants closer follow-up but it is unclear if a more aggressive treatment is needed $[16,17]$.

Even if our findings suggest a prognostic impact of a RV/LV ratio $\geqslant 1.1$ in patients with a sPESI of 0 , the impact of such a finding on the management of these patients remains unclear. AUJESKY et al. [18] found that early outpatient treatment was safe for those with a low-risk PESI, without additional testing. ZoNDAG et al. [19] also did not find an association between RVD by MDCT and a worse prognosis in low-risk patients selected using the Hestia criteria for outpatient treatment. More recently, DEN ExTER et al. [8] found that measuring $\mathrm{N}$-terminal pro-brain natriuretic peptide in low-risk patients using the Hestia criteria was not associated with a decrease in outcome events.

Our choice of primary composite outcome might necessitate further discussion. We included recurrent $\mathrm{PE}$ in the composite in order to be in accordance with most previously published PE prognostic studies. However, RVD could influence more specifically PE-related death or shock but not VTE recurrence and other causes of death. Of note, in our study, all six patients with a composite outcome had at least either PE-related death or shock (supplementary table S2). Therefore, the results remain unchanged if we excluded VTE recurrence from the composite and replace all-cause death by PE-related death.

There are several limitations to our study. First, it is a retrospective analysis of three prospective cohorts, which limits our conclusions. Second, although we could analyse many patients with a sPESI of 0 , the number of events was very low, as expected in this subgroup of low-risk patients. Third, we cannot

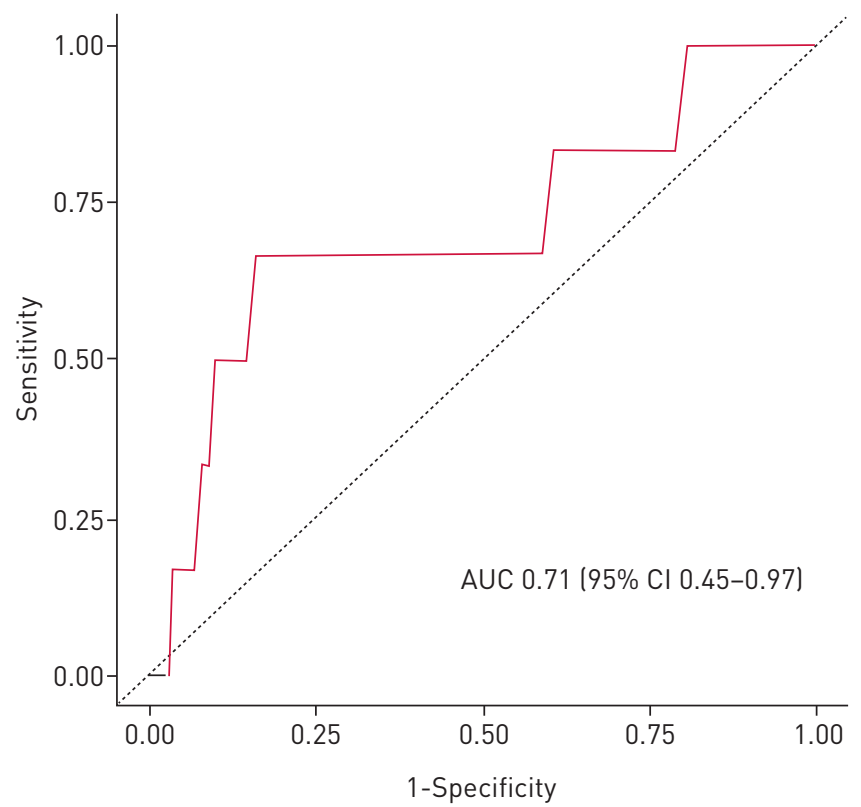

FIGURE 2 Receiver operating characteristic curve for right/left ventricle ratio measured by multidetector computed tomography with regard to 30-day adverse event composite. AUC: area under the curve. 
TABLE 5 Multidetector computed tomography right ventricular dysfunction (RVD)-assessed performance to evaluate the 30-day adverse event composite according to different threshold values

\begin{tabular}{cccccc} 
Sensibility & Specificity & Patients with RVD & $\begin{array}{c}\text { Primary composite } \\
\text { outcome without RVD }\end{array}$ & $\begin{array}{c}\text { Primary composite } \\
\text { outcome with RVD }\end{array}$ & p-value \\
\hline $66.7 \%$ & $46.2 \%$ & $420(54 \%)$ & $0.56 \%$ & $0.95 \%$ & 0.692 \\
$66.7 \%$ & $61.8 \%$ & $299(38 \%)$ & $0.42 \%$ & $1.34 \%$ & 0.211 \\
$66.7 \%$ & $76.1 \%$ & $189(24 \%)$ & $0.34 \%$ & $2.12 \%$ & 0.033 \\
$66.7 \%$ & $82.5 \%$ & $139(18 \%)$ & $0.31 \%$ & $2.88 \%$ & 0.011 \\
$50.0 \%$ & $88.6 \%$ & $91(12 \%)$ & $0.44 \%$ & $3.30 \%$ & 0.024
\end{tabular}

RV: right ventricle; LV: left ventricle.

conclude that our results can apply to extreme RV/LV ratios, since only 139 patients (18\%) had a RV/LV ratio larger than 1.2. The small number of patients with an extreme RV/LV ratio and the low number of outcome events may also explain why the AUC of RV/LV ratio lacked precision with a 95\% CI including 0.5. Fourth, interobserver agreement could not be assessed for the measurements of the RV/LV ratio. However, it has been previously shown that interobserver agreement for the measurement of the RV/LV ratio in the transverse axial plan is good $[11,20]$. Fifth, sPESI score was calculated retrospectively and missing data were assumed to be normal. However, less than $1 \%$ of the needed data for the calculation of sPESI were unavailable.

In conclusion, the current study confirms that RVD assessed by MDCT, using a conventional RV/LV ratio of $\geqslant 0.9$ or $\geqslant 1.0$ as a cut-off, is a common finding in patients with PE and a sPESI of 0 , but does not add significant prognostic information for this low risk population. Nevertheless, physicians should still ask radiologists to measure the MDCT RV/LV ratio in those patients since higher values of the RV/LV ratio might still be associated with an increased risk of PE-related adverse events.

\section{References}

1 Konstantinides SV, Torbicki A, Agnelli G, et al. 2014 ESC guidelines on the diagnosis and management of acute pulmonary embolism. Eur Heart J 2014; 35: 3033-3069, 3069a-3069k.

2 Aujesky D, Obrosky DS, Stone RA, et al. Derivation and validation of a prognostic model for pulmonary embolism. Am J Respir Crit Care Med 2005; 172: 1041-1046.

3 Jiménez D, Aujesky D, Moores L, et al. Simplification of the pulmonary embolism severity index for prognostication in patients with acute symptomatic pulmonary embolism. Arch Intern Med 2010; 170: 1383-1389.

4 Elias A, Mallett S, Daoud-Elias M, et al. Prognostic models in acute pulmonary embolism: a systematic review and meta-analysis. BMJ Open 2016; 6: e010324.

5 Becattini C, Agnelli G, Lankeit M, et al. Acute pulmonary embolism: mortality prediction by the 2014 European Society of Cardiology risk stratification model. Eur Respir J 2016; 48: 780-786.

6 Becattini C, Agnelli G, Germini F, et al. Computed tomography to assess risk of death in acute pulmonary embolism: a meta-analysis. Eur Respir J 2014; 43: 1678-1690.

7 Trujillo-Santos J, den Exter PL, Gómez V, et al. Computed tomography-assessed right ventricular dysfunction and risk stratification of patients with acute non-massive pulmonary embolism: systematic review and meta-analysis. $J$ Thromb Haemost 2013; 11: 1826-1832.

8 den Exter PL, Zondag W, Klok FA, et al. Efficacy and safety of outpatient treatment based on the hestia clinical decision rule with or without $\mathrm{N}$-terminal pro-brain natriuretic peptide testing in patients with acute pulmonary embolism. A randomized clinical trial. Am J Respir Crit Care Med 2016; 194: 998-1006.

9 Sanchez O, Trinquart L, Caille V, et al. Prognostic Factors for Pulmonary Embolism: the PREP study, a prospective multicenter cohort study. Am J Respir Crit Care Med 2010; 181: 168-173.

10 Jiménez D, Lobo JL, Monreal M, et al. Prognostic significance of multidetector CT in normotensive patients with pulmonary embolism: results of the protect study. Thorax 2014; 69: 109-115.

11 Becattini C, Agnelli G, Vedovati MC, et al. Multidetector computed tomography for acute pulmonary embolism: diagnosis and risk stratification in a single test. Eur Heart J 2011; 32: 1657-1663.

12 Robin X, Turck N, Hainard A, et al. pROC: an open-source package for R and S+ to analyze and compare ROC curves. BMC Bioinformatics 2011; 12: 77.

13 Sanchez O, Trinquart L, Colombet I, et al. Prognostic value of right ventricular dysfunction in patients with haemodynamically stable pulmonary embolism: a systematic review. Eur Heart J 2008; 29: 1569-1577.

14 Sanchez O, Trinquart L, Planquette B, et al. Echocardiography and pulmonary embolism severity index have independent prognostic roles in pulmonary embolism. Eur Respir J 2013; 42: 681-688.

15 Barrios D, Rosa-Salazar V, Morillo R, et al. prognostic significance of right heart thrombi in patients with acute symptomatic pulmonary embolism. Chest 2017; 151: 409-416.

16 Barrios D, Rosa-Salazar V, Jiménez D, et al. Right heart thrombi in pulmonary embolism. Eur Respir J 2016; 48: 1377-1385.

17 Barrios D, Chavant J, Jiménez D, et al. Treatment of right heart thrombi associated with acute pulmonary embolism. Am J Med 2017; 130: 588-595. 
18 Aujesky D, Roy P-M, Verschuren F, et al. Outpatient versus inpatient treatment for patients with acute pulmonary embolism: an international, open-label, randomised, non-inferiority trial. Lancet 2011; 378: 41-48.

19 Zondag W, Vingerhoets LMA, Durian MF, et al. Hestia criteria can safely select patients with pulmonary embolism for outpatient treatment irrespective of right ventricular function. J Thromb Haemost 2013; 11: 686-692.

20 Kang DK, Ramos-Duran L, Schoepf UJ, et al. Reproducibility of CT Signs of Right Ventricular Dysfunction in Acute Pulmonary Embolism. Am J Roentgenol 2010; 194: 1500-1506. 